\title{
Energy-aware robust model predictive control based on noisy wireless sensors ${ }^{\star}$
}

\author{
Daniele Bernardini ${ }^{\mathrm{a}, 1}$, Alberto Bemporad ${ }^{\mathrm{b}}$ \\ a Department of Mechanical and Structural Engineering, University of Trento, via Mesiano, 77, 38100 Trento, Italy \\ ${ }^{\mathrm{b}}$ IMT Institute for Advanced Studies Lucca, Piazza S. Ponziano, 6, 55100 Lucca, Italy
}

\section{A R T I C L E I N F O}

\section{Article history:}

Received 7 December 2009

Received in revised form

22 March 2011

Accepted 14 June 2011

Available online 5 October 2011

\section{Keywords:}

Model predictive control

Energy-aware control

Wireless sensors

\begin{abstract}
A B S T R A C T
Wireless sensor networks (WSNs) are becoming fundamental components of modern control systems due to their flexibility, ease of deployment and low cost. However, the energy-constrained nature of WSNs poses new issues in control design; in particular the discharge of batteries of sensor nodes, which is mainly due to radio communications, must be taken into account. In this paper we present a novel transmission strategy for communication between controller and sensors which is intended to minimize the data exchange over the wireless channel. Moreover, we propose an energy-aware control technique for constrained linear systems based on explicit model predictive control (MPC), providing closed-loop stability in the presence of disturbances. The presented control schemes are compared to traditional MPC techniques. The results show the effectiveness of the proposed energy-aware approach, which achieves a profitable trade-off between energy savings and closed-loop performance.
\end{abstract}

(c) 2011 Elsevier Ltd. All rights reserved.

\section{Introduction}

The wireless sensor network (WSN) is an emerging technology for collecting large amounts of measurement data that were previously cost prohibitive. By deploying a large number of cheap, small, and low-consumption sensors equipped with radios, wireless sensing in automation aims at reducing the costs of cabling and their maintenance due to wear and tear. Another advantage of WSNs is the possibility of rapidly reconfiguring the communication infrastructure in case of failures or additions of new components (Akyildiz, Su, Sankarasubramaniam, \& Cayirci, 2002).

On the other hand, compared to standard wired sensors WSNs pose new challenges for control design, such as energy consumption and channel reliability issues. While some interesting work has been done for the latter, such as modeling packet dropouts and addressing time delays (Antsaklis \& Baillieul, 2004; Kumar, 2001; Sinopoli, Sharp, Schenato, Schaffert, \& Sastry, 2003), energyaware control is still a rather open problem. Energy budget is highly constrained to maximize the expected lifetime of battery-operated sensor nodes, therefore preventing frequent cumbersome and expensive maintenance for replacing batteries. These considerations

\footnotetext{
th This work was partially supported by the European Commission under the WIDE project, contract number FP7-IST-224168. This paper was not presented at any IFAC meeting. This paper was recommended for publication in revised form by Associate Editor Martin Guay under the direction of Editor Frank Allgöwer.

E-mail addresses: daniele.bernardini@ing.unitn.it (D. Bernardini), bemporad@imtlucca.it (A. Bemporad).

1 Tel.: +390461 282510; fax: +390577 1698077 .
}

motivate the need of developing new control techniques that, aware of communication and power consumption aspects of the wireless nodes, ensure an optimized controller-sensor operation.

In a wireless device the radio chip is the primary source of energy consumption, hence radio usage must be minimized to achieve a satisfactory network lifetime (Raghunathan, Schurgers, Park, \& Srivastava, 2002; Schurgers, 2002). In recent years some work was done for addressing the energy problem from the point of view of both the communication network and the system architecture, i.e., by proposing consumption-efficient routing protocols (Jones, Sivalingam, Agrawal, \& Chen, 2004; Rajendran, Obraczka, \& Garcia-Luna-Aceves, 2006) or dynamic power management techniques (Akkaya \& Younis, 2005; Raghunathan, Pereira, Srivastava, \& Gupta, 2005).

In this paper we address the issue from the complementary angle of control design. We focus on control systems where feedback is provided by a WSN. Previous works related to this topic include (Walsh \& Hayes, 2007), where a properly tuned control law is designed to achieve real-time regulation of the network transmission rate, and (Quevedo, Ahlén, \& Goodwin, 2008), where a predictive controller is proposed to optimize the trade-off between power transmission and system performance, as a function of the wireless channel estimated reliability. Moreover, communication between controller, sensors and actuators under nominal conditions is addressed in Zhang, Chen, and Chen (2007), where the authors present a network transmission strategy, model the networked plant as a mixed logical dynamical (MLD) system (Bemporad \& Morari, 1999), and formulate a nominal control problem based on mixed-integer programming (MIP). With respect to this work, we wish to avoid the need of online MIP solver to avoid excessive computation complexity, and 
to improve system performance by exploiting two-way channel communications.

We take into account a few key aspects to obtain effective energy savings. First, we consider the energy costs of both transmitting and receiving packets, which are usually very similar (Feeney \& Nilsson, 2001; Moteiv Corporation, 2006). Second, we avoid idle listening as much as possible, i.e., the radio chip of the sensor is completely turned off when no incoming packet is expected (Raghunathan et al., 2002). Finally, as common wireless protocols have a relatively large fixed cost due to communication overheads (Feeney \& Nilsson, 2001), although a single measurement acquisition can be stored in few bytes, the transmission of small packets has a disproportionately high energy price compared to transmitting entire sets of measurements. As a consequence, transmitting $n$ measurements, for small $n$, costs almost as transmitting a single measurement.

By considering the above aspects, we address the energy problem of WSNs together with the optimal control of the global networked process. The main idea is to develop a network transmission strategy where measurements are transmitted to the controller only when necessary, and to design a control scheme aware of that transmission strategy, in order to allow a substantial reduction of radio usage without excessively sacrificing closedloop performance. In our framework we assume the use of wireless sensors within "Class 2-Closed-loop supervisory control" of the taxonomy of the ISA-SP100 standard $^{2}$ where information availability is often not safety-critical. Accordingly, we idealize the wireless communication channel, neglecting packet dropouts and delays, under the assumption that latency and jitter issues (due for instance to packet retransmission after a dropout) are not critical. Taking into account such practical aspects of real wireless networks is beyond the scope of this work; a discussion on the extension of the presented results to the case of real networks with delays and packet loss is given in Section 4.1. A preliminary version of this paper has appeared in Bernardini and Bemporad (2008, 2009).

The paper is organized as follows. We start in Section 2 by describing the wireless communication strategy intended to minimize transmissions. Two cases are addressed: first, in Section 2.1, we consider noiseless state feedback, where a single wireless node, possibly embedding several on-board sensors, is in charge of closing the control loop. Then, in Section 2.2, we extend the approach to the case of uncertain state measurements, where feedback is provided by a wireless network formed by a remote controller and a short range WSN, where several sensors measure the same physical quantities to improve disturbance rejection. For this case, in Section 3 we propose a control solution based on explicit MPC that accounts for the transmission strategy and guarantees convergence and constraints satisfaction. Finally, results of closed-loop simulations are reported in Section 4, and conclusions are drawn in Section 5.

\section{Notation}

In this manuscript, $I_{n}$ is the $n \times n$ identity matrix and $\mathbf{0}_{n}$ is the null vector of $\mathbb{R}^{n}$. Given a vector $x \in \mathbb{R}^{n}$ and a matrix $Q \in \mathbb{R}^{n \times n}, x^{T}$ denotes the transpose of $x, \lambda_{i}(Q), i=1, \ldots, n$, are the eigenvalues of $Q$, and $\|Q x\|_{\infty} \triangleq \max _{i \in\{1, \ldots, n\}}\left|(Q x)_{i}\right|$, where $(Q x)_{i}$ denotes the $i$ th element of $Q x$. Given two sets $\mathcal{A}$ and $\mathscr{B}$, hull $\{\mathcal{A}, \mathscr{B}\}$ is the convex hull of $\mathscr{A} \cup \mathcal{B}, \mathscr{A} \oplus \mathscr{B} \triangleq\{a+b: a \in \mathcal{A}, b \in \mathscr{B}\}$ is the Minkowski sum of $\mathcal{A}$ and $\mathscr{B}$, and $d(x, \mathcal{A}) \triangleq \inf _{y \in \mathcal{A}}\|x-y\|$ is the distance of $x$ form $\mathcal{A} . \mathbb{N}$ is the set of natural numbers including zero.

\footnotetext{
2 ISA-SP100.14 Wireless Networks Optimized for Industrial Monitoring, 2006, http://www.isa.org/filestore/ISASP100_14_CFP_14Jul06_Final(2).pdf.
}

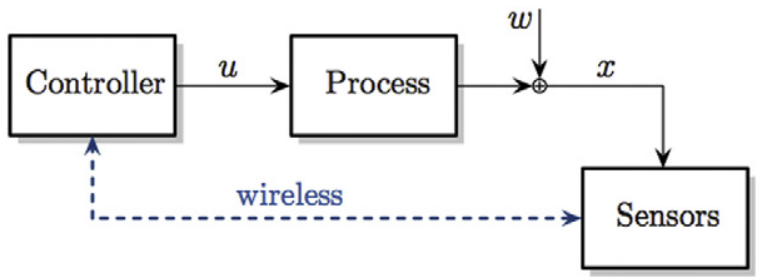

Fig. 1. Control loop scheme with feedback from a single wireless node.

\section{Energy-aware transmission strategy}

\subsection{Exact feedback from a single wireless node}

Consider a control loop where a single node, which possibly embeds several sensors, collects and transmits measurements to a controller through a wireless channel. The controller computes the input signals and delivers them to the actuation device through a wired channel (see Fig. 1). The controlled process is modeled by the discrete-time linear system

$x(k+1)=A x(k)+B u(k)+w(k)$

where $x(k) \in \mathbb{R}^{n_{x}}$ is the state, $u(k) \in \mathbb{R}^{n_{u}}$ is the input, $w(k) \in \mathcal{W}$ is an additive disturbance, and $k \in \mathbb{N}$ is the time index. $W \subset \mathbb{R}^{n_{x}}$ is a given polytope containing the origin. State and input vectors are subject to the constraints

$x \in \mathcal{X}, \quad u \in \mathcal{U}$,

where $\mathcal{X}, \mathcal{U}$ are polyhedra containing the origin in their interior. We assume that full state measurements are collected at every time step by $n_{x}$ sensors embedded in a single wireless node. We define the following transmission strategy: At time step $k$, the wireless sensor node transmits the measurement

$x(k)=\left[x_{1}(k), x_{2}(k), \ldots, x_{n_{x}}(k)\right]^{T}$

to the controller if and only if

$\exists i \in\left\{1,2, \ldots, n_{x}\right\}:\left|x_{i}(k)-\hat{x}_{i}(k)\right|>\varepsilon_{i}$

where $\varepsilon=\left[\varepsilon_{1}, \varepsilon_{2}, \ldots, \varepsilon_{n_{x}}\right]^{T}$ is a vector of threshold values $\varepsilon_{i} \geq 0$ for every component of the state $x$. More compactly, condition (3) can be expressed by using a binary variable $\delta(k)$

$[\delta(k)=1] \leftrightarrow[x(k)-\hat{x}(k) \notin \mathcal{E}]$

where $\mathcal{E} \triangleq\left\{x \in \mathbb{R}^{n_{x}}:\left|x_{i}\right| \leq \varepsilon_{i}, i=1,2, \ldots, n_{x}\right\}$ is the box defined by the threshold vector $\varepsilon$, and $\hat{x}(k)$ is a prediction of the measured value $x(k)$ precalculated by the controller and transmitted beforehand to the wireless node. Predictions are updated in a two-way communication, as follows: when $\delta(k)=1$ the sensor transmits the measurement $x(k)$ to the controller, which computes a set of $M$ updated predictions

$\{\hat{x}(k+j)\}_{j=1}^{M}=\{\hat{x}(k+1), \hat{x}(k+2), \ldots, \hat{x}(k+M)\}$,

and transmits them to the sensor. Moreover, if the controller does not receive any measurement for $M$ time steps, i.e.,

$\delta(k)=\delta(k-1)=\cdots=\delta(k-M+1)=0$,

a one-way communication from controller to sensor node takes place to send $M$ updated predictions $\{\hat{x}(k+j)\}_{j=1}^{M}$, computed using $\hat{x}(k)$ as an estimation of the current state $x(k)$. We refer to $M$ as the estimation horizon and to $\{\hat{x}(k+j)\}_{j=1}^{M}$ as the prediction buffer.

Although the above network transmission strategy is introduced here for linear systems, it is very general and can be implemented in a wide set of scenarios, since the predictions $\hat{x}$ can be calculated with any estimation technique depending on the application at hand. The threshold $\varepsilon$ is an important tuning knob of 


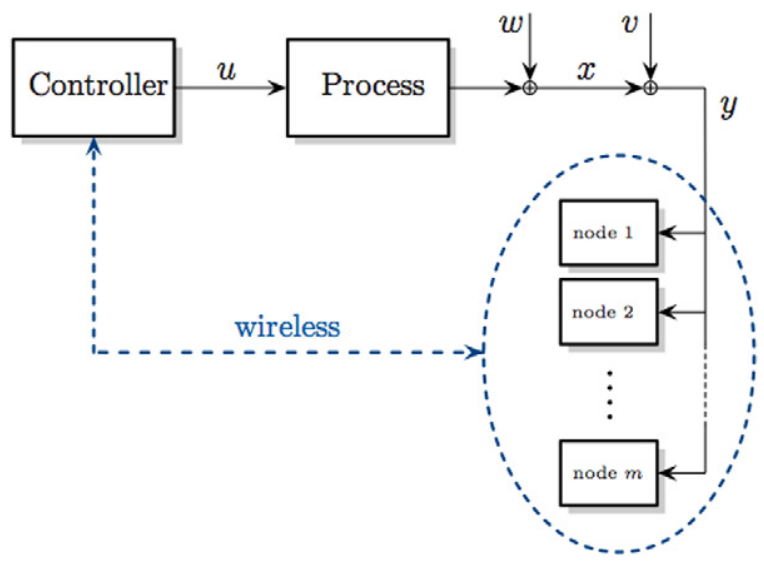

Fig. 2. Control loop scheme with feedback from multiple wireless nodes.

the approach, as it trades off closed-loop performance versus traffic over the wireless channel. Note that the threshold logic (3) allows one to gather information on measured variables even when no measurement is received: if $\delta(k)=0$, then $x(k) \in \varepsilon \oplus$ $\{\hat{x}(k)\}$. In other words, from the controller's point of view a nonmeasurement is a set-valued measurement and, with an opportune choice of $\varepsilon$, this can be usefully exploited in set-membership estimation algorithms, as detailed in Section 2.2.1.

The value of the estimation horizon $M$ must be chosen according to a trade-off between energy consumption and reliability of predictions. In fact, due to the presence of disturbances, the accuracy of the predicted state values decreases with the horizon length, i.e., if $\delta(k)=\delta(k+1)=\cdots=\delta(k+j)=0$, the difference $\left|x_{i}(k+j)-\hat{x}_{i}(k+j)\right|$, in general, is likely to grow with $j \in\{0,1, \ldots, M-1\}$. Hence, an overly large $M$ will lead to unnecessary transmissions of useless far-in-the-future predictions, that will be discarded by the sensor node because of (3).

A notable consequence of the proposed transmission strategy is that idle listening is almost completely avoided. In fact, assuming clock synchronization between the sensor node and the remote controller, the sensor node can wake up its radio chip only when a communication (of updated predictions) is expected to come.

\subsection{Noisy feedback from multiple wireless nodes}

In this section we extend the results of Section 2.1 to the case of noisy state measurements. We consider a control loop where feedback is provided by a wireless network with several sensor nodes, where every node collects a noisy measurement of the state vector (see Fig. 2). Redundancy is exploited here in order to mitigate output measurement errors. Sensor nodes communicate among them using low power (short-range) transmissions, and determine whether to send the measurement to the remote controller (and which value to send) by means of an estimation algorithm.

In the following we consider a system of the form (1) subject to state and input constraints (2), where we remove the hypothesis of exact state feedback. Instead, we assume that measurements are provided by a local WSN of $m$ nodes, indexed by $i=1, \ldots, m$, each one measuring the state vector $x(k)$. The current measurement given by the $i$-th node is defined as

$y^{i}(k)=x(k)+v^{i}(k)$

where $v^{i} \in \mathcal{V}^{i}$ is an unknown but bounded disturbance, and

$\mathcal{V}^{i}=\left\{v \in \mathbb{R}^{n_{x}}:\left|v_{j}\right| \leq v_{j, \max }^{i}, j=1, \ldots, n_{x}\right\}$.
The vectors $v_{\max }^{i}=\left[v_{1, \max }^{i}, \ldots, v_{n_{x} \text { max }}^{i}\right], i=1, \ldots, m$, are assumed to be known to every node. We define the transmission strategy as follows.

At time step $k$ let $h=1+\left(\left\lfloor\frac{k}{n}\right\rfloor \bmod m\right)$. We refer to the $h$-th node as the master node. The positive integer parameter $n$ represents the number of consecutive time steps for which the master node does not change. A short range communication transmitting the value $y^{i}(k)$ takes place from all the nodes $i \neq h$, called slave nodes, to the master node. Once all the measurements are delivered, the master node performs a simple set-membership estimation by computing the box set

$$
\begin{aligned}
y(k) & =\bigcap_{i=1}^{m} y^{i}(k) \\
& =\left\{x: b_{j, \min } \leq x_{j} \leq b_{j, \max }, j=1, \ldots, n_{x}\right\}
\end{aligned}
$$

where

$\begin{aligned} b_{j, \min } & =\max _{i \in\{1, \ldots, m\}} y_{j}^{i}(k)-v_{j, \max }^{i} \\ b_{j, \max } & =\min _{i \in\{1, \ldots, m\}} y_{j}^{i}(k)+v_{j, \max }^{i}\end{aligned}$

and

$y^{i}(k)=\left\{x:\left|y^{i}(k)-x\right| \leq v_{\max }^{i}\right\}, \quad i=1, \ldots, m$,

are all the feasible sets of states according to each node's measurements. Finally, the master node transmits $y(k)$ to the controller through a long range wireless communication if and only if there exists $j \in\left\{1, \ldots, n_{x}\right\}$ such that

$\left[b_{j, \min }-\hat{x}_{j}(k)<-\varepsilon_{j}\right] \vee\left[b_{j, \max }-\hat{x}_{j}(k)>\varepsilon_{j}\right]$

where $\hat{x}(k) \in \mathbb{R}^{n_{x}}$ is a prediction of the current state value $x(k)$, as in Section 2.1. Condition (9) is equivalent to

$y(k) \nsubseteq \mathscr{E} \oplus\{\hat{x}(k)\}$

where $\mathcal{E}=\left\{x:\left|x_{j}\right| \leq \varepsilon_{j}, j=1, \ldots, n_{x}\right\}$. We represent the transmission condition $(10)$ with $[\delta(k)=1]$, where $\delta$ is a binary variable.

Note that the predictions $\hat{x}$ are exploited only by the current master node. Let $h_{k}=1+\left(\left\lfloor\frac{k}{n}\right\rfloor \bmod m\right)$ and $h_{k-1}=1+$ $\left(\left\lfloor\frac{k-1}{n}\right\rfloor \bmod m\right)$. At time $k$, if $h_{k} \neq h_{k-1}$, then the $h_{k-1}$-th node (the previous master) is required to transmit, along with the measurement, also the prediction buffer to the $h_{k}$-th node (the current master). We adopt a time-varying master node in order to distribute long-range transmissions among all the sensor nodes, so to have uniform battery discharge. Other scheduling policies may be possible, for instance in case only one node is equipped with a communication device for the wide-area network.

Here we assume that the $m$ nodes are spatially close to each other, so that the energy cost for a short range transmission is very small with respect to a wide-area transmission, and the communication activity between sensor nodes can be neglected. However, it is easy to extend the approach by using a threshold logic also for local transmissions, and having slave sensor nodes send the measurements to the master only when they are sufficiently far from a predicted value.

\subsubsection{State estimation algorithm}

Since the additive disturbance $v$ affects the output (4) at every time step, an exact state measurement is unavailable either if the measurements have been transmitted or not. In the following we present an algorithm to obtain an estimation of the true state value, needed to compute the control action. 
Due to the transmission strategy (3), the state $x(k)$ is always subject to a known set-membership relation, regardless of the master node's decision to forward the data. In particular, we have

$x(k) \in \begin{cases}y(k) & \text { if } \delta(k)=1 \\ \mathscr{E} \oplus\{\hat{x}(k)\} & \text { otherwise. }\end{cases}$

Hence, in the absence of packet dropouts, information on the measured variables is gathered even when no feedback is provided (i.e., when $\delta(k)=0$ ). We propose to use an algorithm based on set-membership estimation (Milanese \& Vicino, 1991), which takes into consideration (11) to reduce the uncertainty on the state.

Let $\mathcal{Z}(k \mid k)$ and $\mathcal{Z}(k+1 \mid k)$ be the sets of all the possible values of the state $x$ at time $k$ and $k+1$, respectively, given the feedback information at time $k$. The set $\mathcal{Z}(k+1 \mid k)$ is defined by the prediction step

$\mathcal{Z}(k+1 \mid k)=A Z(k \mid k) \oplus B\{u(k)\} \oplus W$

and the set $\mathcal{Z}(k \mid k)$ is obtained with the correction step

$\mathcal{Z}(k \mid k)= \begin{cases}\mathcal{Z}(k \mid k-1) \cap \mathcal{Y}(k) & \text { if } \delta(k)=1 \\ \mathbb{Z}(k \mid k-1) \cap(\mathscr{E} \oplus\{\hat{x}(k)\}) & \text { otherwise. }\end{cases}$

The estimation $\bar{x}(k \mid k)$ of the actual state $x(k)$, given the feedback information at time $k$, is defined as the centroid $c$ of $Z(k \mid k)$, computed as

$\bar{x}(k \mid k)=c(\mathcal{Z}(k \mid k)) \triangleq \frac{1}{n_{z}} \sum_{i=1}^{n_{z}} z_{i}$

where $n_{z}$ is the number of the vertices $z_{1}, z_{2}, \ldots, z_{n_{z}}$ of $\mathcal{Z}(k \mid k)$.

This set-membership algorithm can lead to very complex representations of $\mathcal{Z}$ (i.e., a high number of vertices). In order to lower the computational burden and preserve the implementability of the control scheme, in the simulations presented in Section 4 we use a sub-optimal estimation algorithm derived from Chisci, Garulli, and Zappa (1996), where outbounding parallelotopes are used to approximate the actual state set $\mathcal{Z}(k \mid k)$.

\section{MPC control design}

In this section we present a robust control scheme based on explicit MPC and min-max optimization, suitable for handling the wireless transmission strategy proposed in Section 2. We focus on the multiple-node setup addressed in Section 2.2 for brevity reasons, providing in Remark 6 comments on how to derive analogous results for the single node case.

Motivated by the search for a good trade-off between closedloop performance and network transmission rate, we want to design a robust controller for system (1), which guarantees convergence of the state to the origin despite the disturbances affecting the system. In the case of noisy state measurements we have to deal with three sources of uncertainty: the additive disturbance $w$, the feedback error due to the transmission strategy (9), and the measurement noise $v^{i}, i=1, \ldots, m$. Due to the presence of these persistent disturbances the state cannot be directly regulated to the origin. Therefore, following the idea of dual mode MPC (Scokaert \& Mayne, 1998), we set up an outer control mode, which drives the state $x$ to a given set $X_{0}$, and an inner control mode which robustly keeps the state in $x_{0}$. The set $X_{0}$ is assumed to be robust positively invariant with respect to additive disturbances, as from the following definition (Blanchini, 1999; Blanchini \& Miani, 2008).

Definition 1. The set $\mathcal{X}_{0} \subseteq \mathbb{R}^{n}$ is robust positively invariant (RPI) for a system of the form (1) if and only if $\forall x(0) \in \mathcal{X}_{0}$ and $\forall w(k) \in$ $W$ the solution $x(k) \in X_{0}, \forall k \in \mathbb{N}$.

\subsection{Inner mode}

The inner control mode is based on a switching feedback control law, defined in the following lemma.
Lemma 2. Let $K \in \mathbb{R}^{n_{u} \times n_{x}}$ and $A_{c} \triangleq A+B K$ be such that $\left|\lambda_{i}\left(A_{c}\right)\right|<$ $1, \forall i=1, \ldots, n_{x}$. Let $f(k)$ be an unknown but bounded disturbance such that

$f(k) \in \mathcal{W} \oplus\{-B K \operatorname{hull}\{\mathcal{E}, \mathcal{V}\}\}$

with $\mathcal{V} \triangleq \bigcap_{i=1}^{m} \mathcal{V}^{i}$. Let $\mathcal{X}_{0} \subseteq \mathcal{X}$ be an RPI set for the system

$x(k+1)=A_{c} x(k)+f(k)$

such that $K X_{0} \subseteq U$. Let $x$ be the state of (1)-(2) and (4) receiving feedback according to (9), in closed-loop with

$u(k)=K \bar{x}(k \mid k)$

where $\bar{x}(k \mid k)$ is defined as in (14). If $x(k) \in X_{0}$, then $x(k+t) \in X_{0}$, for all $t \in \mathbb{N}$.

Proof. By substituting (17) in (1) we obtain

$x(k+1)=A x(k)+B K \bar{x}(k \mid k)+w(k)$.

The combination of (4) and (5) with the transmission logic (9) and with the state estimation equations (12)-(14) imply

$x(k)-\bar{x}(k \mid k) \in \begin{cases}\mathcal{V} & \text { if } \delta(k)=1 \\ \mathcal{E} & \text { otherwise }\end{cases}$

and, conservatively,

$x(k)-\bar{x}(k \mid k) \in \operatorname{hull}\{\mathscr{E}, \mathcal{V}\}$

for all $\delta(k) \in\{0,1\}$. Combining (18) and (20) we have

$x(k+1) \in\left\{A_{c} x(k)\right\} \oplus \mathbb{W} \oplus\{-B K(x(k)-\bar{x}(k \mid k))\}$

$\in\left\{A_{c} x(k)\right\} \oplus \mathcal{W} \oplus\{-B K \operatorname{hull}\{\mathcal{E}, \mathcal{V}\}\}$.

Using (21) and (15), we have that system (18) is overapproximated by (16), which is nominally stable by hypothesis. Being $\mathcal{X}_{0}$ RPI for (16), it is RPI also for (18). Fulfillment of constraints (2) follows by $\mathcal{X}_{0} \subseteq \mathcal{X}$ and $K \mathcal{X}_{0} \subseteq \mathcal{U}$

We can use known results on RPI sets for linear systems to design $X_{0}$, see e.g. (Blanchini, 1999; Blanchini \& Miani, 2008; Kolmanovsky \& Gilbert, 1998; Scokaert \& Mayne, 1998).

\subsection{Outer mode}

To design the outer control mode we propose an algorithm based on explicit model predictive control. MPC is widely spread in industry for control design of highly complex multivariable processes under constraints on input and state variables (Camacho \& Bordons, 2004; Maciejowski, 2002; Qin \& Badgwell, 2003). The idea behind MPC is to solve at each sampling time an open-loop finite-horizon optimal control problem based on a given prediction model of the process, by taking the current state of the process as the initial state. Only the first sample of the sequence of future optimal control moves is applied to the process. At the next time step, the remaining moves are discarded and a new optimal control problem based on new measurements is solved over a shifted prediction horizon.

An alternative approach to evaluate the MPC law was proposed in Bemporad, Morari, Dua, and Pistikopoulos (2002): rather then solving the optimization problem on-line for the current state vector, by employing techniques of multiparametric programming the problem is solved off-line for all state vectors within a given range, providing the explicit dependence of the control input on the state and reference, which is piecewise affine (PWA) and continuous (for a survey on explicit MPC the reader is referred to Alessio \& Bemporad, 2008; for industrial applications see, e.g., Di Cairano \& Tseng, 2010). 
In our framework, an explicit formulation of MPC is a natural choice for many reasons: primarily, it can handle constraints and can be formulated to achieve robust control in the presence of disturbances. Moreover, it allows the cheap computation of the prediction buffer $\{\hat{x}(k+j)\}_{j=1}^{M}$, by evaluating the future evolution of a simple closed-loop PWA system. We present a scheme derived from min-max MPC (Bemporad, Borrelli, \& Morari, 2003; Kerrigan \& Maciejowski, 2003; Scokaert \& Mayne, 1998), where the goal is to steer the state to a target set while minimizing a performance index over the worst-case disturbance realization. The basic idea of our approach is to include the knowledge of the transmission strategy (9) in the MPC optimization problem, so that the dynamics of the predicted state $\hat{x}$ can be coherently modeled into the optimizer.

Let $\left\{w_{k+j \mid k}^{\ell}\right\}_{j=0}^{N-1}$ denote the $\ell$-th sequence of disturbance realizations over a prediction horizon of $N$ steps given the state estimation at time $k$, with $\ell \in \mathcal{L}$ (for simplicity of notation, since now on we will drop the subscript ${ }_{j=0}$ and superscript ${ }^{N-1}$ ). Further, let $\left\{u_{k+j \mid k}^{\ell}\right\}$ denote the sequence of control moves associated with the $\ell$-th realization, and $\left\{x_{k+j \mid k}^{\ell}\right\}$ the corresponding predicted state sequence. By ignoring the transmission strategy and assuming exact state feedback at every sampling instant, the min-max MPC problem at time $k$ is expressed as in Scokaert and Mayne (1998)

$$
\begin{aligned}
& \min _{\left\{u_{k+j \mid k}^{\ell} \max _{\ell \in \mathcal{L}}\right.} \sum_{j=0}^{N-1} L\left(x_{k+j \mid k}^{\ell}, u_{k+j \mid k}^{\ell}\right) \\
& \text { s.t. } x_{k+j+1 \mid k}^{\ell}=A x_{k+j \mid k}^{\ell}+B u_{k+j \mid k}^{\ell}+w_{k+j \mid k}^{\ell}, \\
& x_{k+j \mid k}^{\ell} \in \mathcal{X}, \\
& u_{k+j \mid k}^{\ell} \in U, \\
& x_{k+N \mid k}^{\ell} \in X_{T}, \\
& x_{k+j \mid k}^{\ell_{1}}=x_{k+j \mid k}^{\ell_{2}} \Rightarrow \quad u_{k+j \mid k}^{\ell_{1}}=u_{k+j \mid k}^{\ell_{2}}, \\
& j=0, \ldots, N-1, \forall \ell, \ell_{1}, \ell_{2} \in \mathcal{L},
\end{aligned}
$$

where $N$ is the control horizon, (22b) is the dynamics equation, (22c)-(22d) are the state and input constraints, (22e) is the target set constraint, and (22f) is the causality constraint, which enforces a single control input for each state, reducing the freedom on the control sequence and making the control law independent of the path taken to reach that state.

Assumption 3. The stage cost $L(x, u)$ is convex over $\mathcal{X} \times \mathcal{u}$ and such that

$L(x, K x) \leq L(y, u), \quad \forall x \in X_{T}, \forall y \notin X_{T}, \forall u \in U$

$L(x, u) \geq \alpha\left(d\left(x, \mathcal{X}_{T}\right)\right), \quad \forall x \notin \mathcal{X}_{T}, \forall u \in U$

where $\alpha$ is a $\mathcal{K}$-function.

The following lemma shows constructively how Assumption 3 can be satisfied.

Lemma 4. Let $\mathcal{X}_{0}=\left\{x \in \mathbb{R}^{n_{x}}: A_{0} x \leq b_{0}\right\}, A_{0} \in \mathbb{R}^{n_{r} \times n_{x}}, b_{0} \in \mathbb{R}^{n_{r}}$. For $i=1,2, \ldots, n_{r}$, let

$$
\begin{gathered}
c_{0, i}=\min _{x, s} s \\
\text { s.t. } s \geq\left\|Q_{x} x\right\|_{\infty}, \\
A_{0}^{i} x=b_{0}^{i},
\end{gathered}
$$

and define

$$
c_{0}=\min _{i \in\left\{1,2, \ldots, n_{r}\right\}} c_{0, i}
$$

with $A_{0}^{i}$ the $i$-th row of $A_{0}$ and $b_{0}^{i}$ the $i$-th element of $b_{0}$. Then the set

$x_{T} \triangleq\left\{x \in \mathbb{R}^{n_{x}}:\left[\begin{array}{c}Q_{x} \\ -Q_{x}\end{array}\right] x \leq c_{0}\left[\begin{array}{l}1 \\ 1\end{array}\right]\right\}$

is such that $\mathcal{X}_{T} \subseteq \mathcal{X}_{0}$, and the stage cost

$L(x, u)=\left\|Q_{x} x\right\|_{\infty}$

satisfies Assumption 3.

Proof. By (24)-(26) it follows that $X_{T}$ is the largest level set of $\left\|Q_{x} x\right\|_{\infty}$ such that $\mathcal{X}_{T} \subseteq \mathcal{X}_{0}$. By construction, $\forall y \notin \mathcal{X}_{T},\left\|Q_{x} y\right\|_{\infty}>$ $c_{0}$, since

$\exists i \in\left\{1,2, \ldots, n_{x}\right\}:\left(Q_{x}^{i} y>c_{0}\right) \vee\left(Q_{x}^{i} y<-c_{0}\right)$

where $Q_{x}^{i}$ is the $i$-th row of $Q_{x}$, which proves (23a). Take $d\left(y, X_{T}\right)=$ $\inf _{x \in X_{T}}\left\|Q_{x}(y-x)\right\|_{\infty}$. As $0 \in \mathcal{X}_{T}$, by definition of inf, $d\left(y, \mathcal{X}_{T}\right) \leq$ $\left\|Q_{x}(y-0)\right\|_{\infty}$. By letting $\alpha(\phi)=\phi$, (23b) follows. Hence, Assumption 3 is satisfied.

Note that the stage cost (27) is only used in the outer mode: as soon as $x(k)$ enters $X_{0}$, a constant feedback control loop of the form $u(k)=K \bar{x}(k)$ is applied, where the gain $K$ can be designed with arbitrary (yet stabilizing) performance criteria.

Under the hypothesis of exact state feedback, the input sequence resulting from the receding horizon solution of problem (22) ensures the asymptotical convergence of the state $x$ of (1) to the target set $\mathcal{X}_{T}$ (see Scokaert \& Mayne, 1998). By solving $N$ mpLPs as in Bemporad et al. (2003), and by using $L(x, u)=\left\|Q_{x} x\right\|_{\infty}$, this solution is obtained in state-feedback piecewise affine form

$u^{*}(x)=F_{i} x+g_{i} \quad$ if $x \in \mathcal{R}_{i}$

where $F_{i} \in \mathbb{R}^{n_{u} \times n_{x}}, g_{i} \in \mathbb{R}^{n_{u}}$ and $\mathcal{R}_{i}=\left\{x \in \mathbb{R}^{n_{x}}: C_{i} x \leq d_{i}\right\}$, with $i \in \ell \triangleq\{1,2, \ldots, r\}$.

Now we need to reintroduce the proposed transmission strategy (9) and the output noise (4). In order to do so, consider the feedback control law derived from (28) and computed on the estimated state $\bar{x}(k \mid k)$ rather than on the true state $x(k)$, that is

$u(k)=F_{j} \bar{x}(k \mid k)+g_{j}$

where $\bar{x}(k \mid k) \in \mathcal{R}_{j}$. Now, let $x(k) \in \mathcal{R}_{i}$. The difference between the optimal input obtained by (28) with full state knowledge and the input (29) actually applied to the system is

$u(k)-u^{*}(x(k))=F_{j} \bar{x}(k \mid k)+g_{j}-F_{i} x(k)-g_{i}$.

Hence, the dynamics of system (1)-(4) in closed-loop with (29) can be recast as

$x(k+1)=\left(A+B F_{i}\right) x(k)+B g_{i}+w(k)+q(k)$

where

$q(k)=B\left(F_{j} \bar{x}(k \mid k)+g_{j}-F_{i} x(k)-g_{i}\right)$.

The quantity $q(k)$ models the input error made with respect to the optimal trajectory obtained with (28), caused by the nonlinear transmission policy and the measurement noise. We consider $q(k)$ as an additional unknown but bounded disturbance within a set $\mathcal{Q}$, with

$$
\begin{gathered}
\mathcal{Q} \triangleq\left\{q \in \mathbb{R}^{n_{x}}: q=B\left(F_{j} \bar{x}+g_{j}-F_{i} x-g_{i}\right),\right. \\
\left.x, \bar{x} \in \mathbb{R}^{n_{x}}, x-\bar{x} \in \operatorname{hull}\{\mathcal{E}, \mathcal{V}\}\right\} .
\end{gathered}
$$

Our goal is to obtain a robust control law with respect to both $w$ and $q$. We cannot directly set up a multiparametric optimization problem including $q$, since the polytope $\mathcal{Q}$ is dependent on $\left\{F_{i}\right\}_{i \in \ell}$ and $\left\{g_{i}\right\}_{i \in \ell}$ which are nonlinear functions of $x$. To overcome this issue, we propose an iterative algorithm: At every step $h$ of the algorithm, the estimated set $Q^{h}$, the gains $\left\{F_{i}\right\}_{i \in \ell}^{h},\left\{g_{i}\right\}_{i \in \ell}^{h}$ and the 
partition $\left\{\mathcal{R}_{i}\right\}_{i \in \ell}^{h}$ are computed as a function of the previous set $Q^{h-1}$. This procedure, to be executed off-line, is defined in Algorithm 1 and is based on the linear system

$x(k+1)=A x(k)+B u(k)+w(k)+q(k)$

and the associated min-max MPC problem

$$
\begin{aligned}
& \min _{\left\{u_{k+j \mid k}^{\ell}\right\}} \max _{\ell \in \mathcal{L}} \sum_{j=0}^{N-1} L\left(x_{k+j \mid k}^{\ell}, u_{k+j \mid k}^{\ell}\right) \\
& \text { s.t. (22c), (22d), (22e), (22f), (33), }
\end{aligned}
$$

together with its explicit solution in state feedback form.

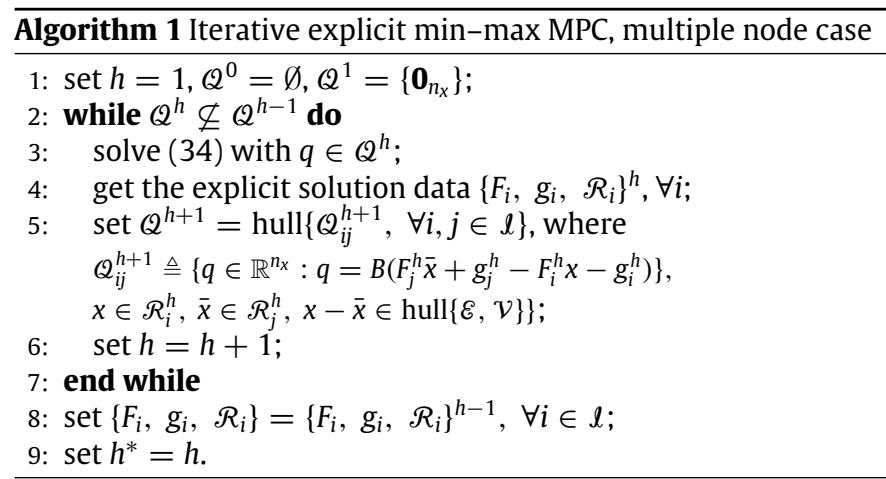

The computation of $\mathcal{Q}$ may be conservative, in the sense that it may return a set larger than necessary. In particular, the controller obtained with Algorithm 1 is robust with respect to all disturbances in $\mathcal{Q}^{h^{*}-1}$, but the actual disturbance realizations are $q(k) \in \mathcal{Q}^{h^{*}} \subseteq \mathcal{Q}^{h^{*}-1}$. Indeed, this does not have any impact on the communication side (and on transmissions savings), and may only affect performance of the control action in the outer control mode. In Section 4 we provide numerical details about the overapproximation of $\mathcal{Q}$ computed on the presented example.

Note that since the actual value of the state $x(k)$ is not known, at time $k$ the controller is required to switch from outer mode to inner mode if and only if

$\mathcal{Z}(k \mid k) \subseteq X_{0}$

which ensures that $x(k) \in X_{0}$. Moreover, since the terminal set $X_{0}$ is constructed to be RPI with respect to the closed-loop system, we can reduce conservativeness in the estimation algorithm by using the prediction step

$\mathcal{Z}(k+1 \mid k)=(A \mathcal{Z}(k \mid k) \oplus B\{u(k)\} \oplus \mathcal{W}) \cap \mathcal{X}_{0}$

instead of (12), if at time $k$ the inner control mode is active.

We can finally define the Robust Energy-Aware MPC with Noisy measurements (REAN-MPC) control scheme in Algorithm 2 and state its properties in the following theorem.

Theorem 5. Let Algorithm 1 admit a solution. Then the state $x$ of (1)-(4) receiving feedback according to (9) and controlled by REANMPC converges asymptotically to the target set $X_{T}$ while satisfying constraints (2). If $\mathcal{Z}(k \mid k) \subseteq \mathcal{X}_{0}$ at any time step $k$, then $x(k+t) \in \mathcal{X}_{0}$ for all $t \in \mathbb{N}$.

Proof. The outer mode explicit control law defined by Algorithms 1-2 is designed to be robust with respect to the additive disturbance $w$ and the feedback error $q$, induced by the measurement noise (4) and the transmission strategy (9). Moreover, by Lemma 4, $x \in \mathcal{X}_{T}$ if and only if $\left\|Q_{x} x\right\|_{\infty} \leq c_{0}$. Then, $\forall x(k) \notin X_{T}, \forall \ell \in$ $\mathcal{L},\left\|Q_{x} x_{k \mid k}^{\ell}\right\|_{\infty}-\left\|Q_{x} x_{k+N \mid k}^{\ell}\right\|_{\infty} \geq 0$. Hence, the outer mode stage cost can be shown to be non-increasing in time, and the proof of

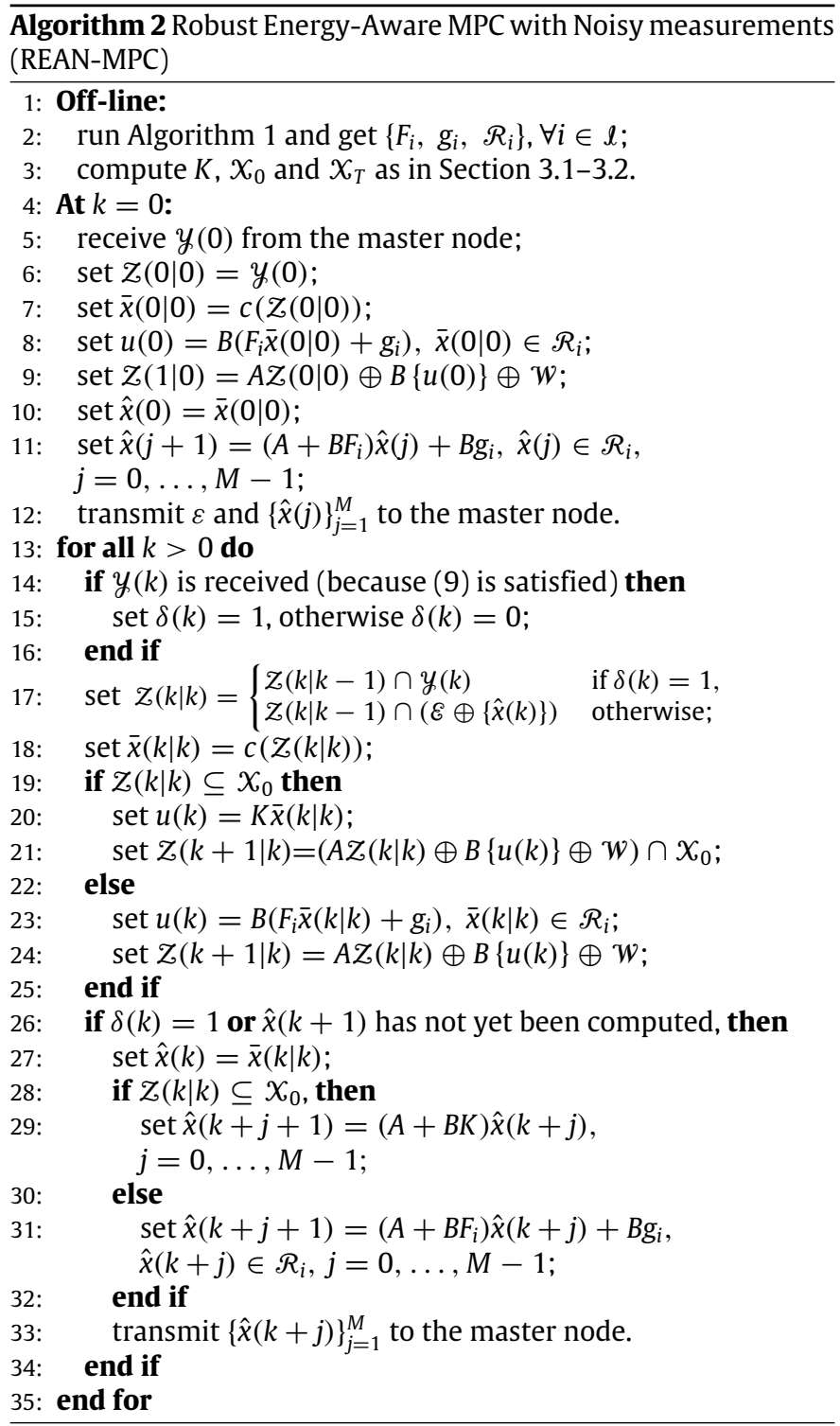

asymptotical convergence to $X_{T}$ follows in a similar fashion of Theorem in Scokaert and Mayne (1998). Once the controller switches to the inner control mode, because (35) is satisfied, robust invariance of closed-loop trajectories with respect to $X_{0}$ follows by Lemma 2.

Remark 6. An energy-aware controller for the case of exact state feedback described in Section 2.1 can be derived in a similar fashion, by taking the output noise set $\mathcal{V}=\emptyset$ and the estimated state

$\bar{x}(k \mid k)= \begin{cases}x(k) & \text { if } \delta(k)=1 \\ \hat{x}(k) & \text { otherwise. }\end{cases}$

The transmission strategy for the single node case needs to be slightly modified in order to deal with the proposed dual mode MPC. In addition to (3), the sensor node is required to transmit the measurements when the state $x(k)$ and its prediction $\hat{x}(k)$ lie in different control mode sets, i.e.,

$$
\begin{aligned}
& {[\delta(k)=0] \leftrightarrow[x(k)-\hat{x}(k) \in \mathcal{E}]} \\
& \quad \wedge\left[\left[x(k) \in X_{0}, \hat{x}(k) \in X_{0}\right] \vee\left[x(k) \notin X_{0}, \hat{x}(k) \notin X_{0}\right]\right]
\end{aligned}
$$

to avoid erroneous switches from outer to inner mode. This controller, referred to as Robust Energy-Aware MPC (REA-MPC), will be considered for comparison purposes in Section 4 . 


\section{Simulation results}

To evaluate the performance of the proposed techniques we consider the open-loop unstable system given by (1) and (2), with

$A=\left[\begin{array}{cc}0.21 & -0.39 \\ -0.39 & 0.82\end{array}\right], \quad B=\left[\begin{array}{l}0 \\ 1\end{array}\right]$,

$\mathcal{X}=\left\{x \in \mathbb{R}^{2}:\left|x_{i}\right| \leq 2, i=1,2\right\}, u=\{u \in \mathbb{R}:|u| \leq$ $1\}, \mathcal{W}=\left\{w \in \mathbb{R}^{2}:\left|w_{i}\right| \leq 0.08, i=1,2\right\}$, and threshold vector $\varepsilon=[0.1,0.1]^{T}$. We assume that the output noise is defined by (4)-(5) with $v_{\max }^{i}=[0.06,0.06]^{T}, \forall i$.

The presented energy-aware control schemes have been compared to "traditional" robust MPCs, which do not take into account the minimization of transmissions over the wireless channel. In this case, no predictions are sent to the sensor nodes and the collected measurements are simply transmitted to the controller at every time step, regardless of the threshold logic. We refer to R-MPC as the robust MPC controller in the exact state feedback framework, and to RN-MPC as the robust MPC controller in the case of noisy measurements. Note that R-MPC and RNMPC algorithms are special cases of REA-MPC and REAN-MPC, respectively, where $\varepsilon=\emptyset$ and $M=0$.

We have run $N_{s}=100$ simulations of $T=10$ time steps each, randomly choosing the initial states $x(0) \in \mathcal{X}$. The weight matrices used to compute the outer and the inner controllers are $Q_{x}=I_{2}, Q_{u}=0.1$. The control horizon is $N=5$ and the estimation horizon is $M=10$. By running Algorithm 1, we have obtained an explicit control law for the outer controller, defined over 7 polyhedral partitions of the state space. This procedure yielded a slightly overapproximated set $\mathcal{Q}$, being

$\mathcal{Q}^{h^{*}}=\left\{q \in \mathbb{R}^{2}: q_{1}=0,\left|q_{2}\right| \leq 0.1533\right\}$,

$\mathcal{Q}^{h^{*}-1}=\left\{q \in \mathbb{R}^{2}: q_{1}=0,\left|q_{2}\right| \leq 0.1695\right\}$.

We consider two quantities to compare the performance of the energy-aware schemes: the rate of data transmission over the wireless channel, assuming equal power consumption in transmitting and receiving packets as usual for short range wireless nodes (Moteiv Corporation, 2006), and the cumulated cost function

$J_{\text {exp }}^{i} \triangleq \sum_{k=1}^{T}\left(\left\|Q_{x} x^{i}(k)\right\|_{\infty}+\left\|Q_{u} u^{i}(k)\right\|_{\infty}\right)$

where $i$ indexes the $i$-th simulation. Simulation results are detailed in Table 1, where

$J_{\text {avg }} \triangleq \frac{1}{N_{S}} \sum_{i=1}^{N_{S}} J_{\text {exp }}^{i}$

is the average experimental performance and $T x$ is the overall transmission rate. REA and REAN controllers achieve a good tradeoff between performance and transmission rate: in the presented simulations REA-MPC grants a reduction in radio utilization by $49.2 \%$, with a $2.5 \%$ loss in the experimental cost function with respect to R-MPC. Six-node REAN-MPC obtains similar results, with a $57.9 \%$ saving in transmissions and a loss of $2.6 \%$ in performance compared to RN-MPC.

In order to further evaluate the sensitivity to noise of the REANMPC setup, Table 1 also reports numerical results for varying number of sensor nodes $m \in\{3,6,9,12\}$. As expected, using more nodes increases transmission savings, at the cost of some performance deterioration.

Moreover, we included in the tests a formulation of R-MPC and of RN-MPC where the sampling time is twice as large. These controllers, referred to in the table as half-rate MPCs, involve $50 \%$ less communications between nodes by simply running at half speed. Results show that the energy-aware controllers obtain
Table 1

Energy-aware MPC: simulation results.

\begin{tabular}{llr}
\hline & $J_{\text {avg }}$ & $T x(\%)$ \\
\hline Exact measurements & & \\
R-MPC & 2.4027 & 100.0 \\
Half-rate R-MPC & 2.5852 & 50.0 \\
REA-MPC & 2.4626 & 50.8 \\
Noisy measurements & & \\
RN-MPC with 6 nodes & 2.5025 & 100.0 \\
Half-rate RN-MPC with 6 nodes & 2.6787 & 50.0 \\
REAN-MPC with 3 nodes & 2.5331 & 77.6 \\
REAN-MPC with 6 nodes & 2.5514 & 51.8 \\
REAN-MPC with 9 nodes & 2.5665 & 45.4 \\
REAN-MPC with 12 nodes & 2.5682 & 43.0 \\
\hline
\end{tabular}

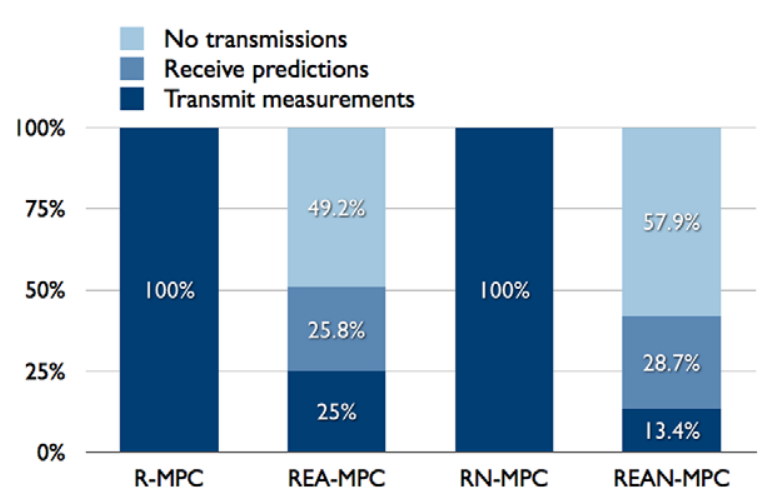

Fig. 3. Average traffic over the wireless channel.

transmission savings comparable to half rate MPCs, providing in addition a better performance and constraints handling (note that half-rate schemes enforce robust constraints only at half time steps).

A comparison of the average data exchange over the wireless channel for the different control schemes is shown in Fig. 3. We can see that with the proposed energy-aware approach a relevant part $(50 \%-70 \%)$ of the total wireless communications is spent to deliver updated predictions to the sensor nodes. In some frameworks, e.g. when the calculation of the control law does not require external information and nodes have sufficient computation capabilities, it could be possible to let the predictions be computed locally by the sensor nodes. In this way one would avoid the need of having transmissions from the controller, dramatically cutting the overall radio power consumption further.

All the tested energy-aware control schemes provide satisfaction of state and input constraints at all time steps. As expected, the input command often saturates to the limit values $\{-1,1\}$ but constraints are always fulfilled, as shown in Fig. 4 for the case of REAN-MPC with 6 sensor nodes. Analogous plots related to other controllers are similar and are omitted for brevity reasons.

Robust control schemes based on min-max optimization problems like those presented in this paper can lead to conservative control action sometimes, since stability with respect to every possible disturbance realization sequence is required. In such cases nominal controllers can be adopted, which in general provide less conservative system performance at the expenses of lack of stability and constraint fulfillment properties. In particular, in the energy-aware framework of this paper, it is easy to derive a nominal counterpart of REAN-MPC by solving (34) with $\mathcal{W}=\mathcal{Q}=\left\{\boldsymbol{0}_{n_{x}}\right\}$ and $X_{T}=\mathbb{R}^{n_{x}}$. Simulations involving nominal energy-aware control schemes are omitted here and are reported in Bernardini and Bemporad (2008, 2009).

\subsection{Discussion on network model}

In the following we revise the assumptions made on the wireless network model (i.e., no delay nor packet loss can occur) 

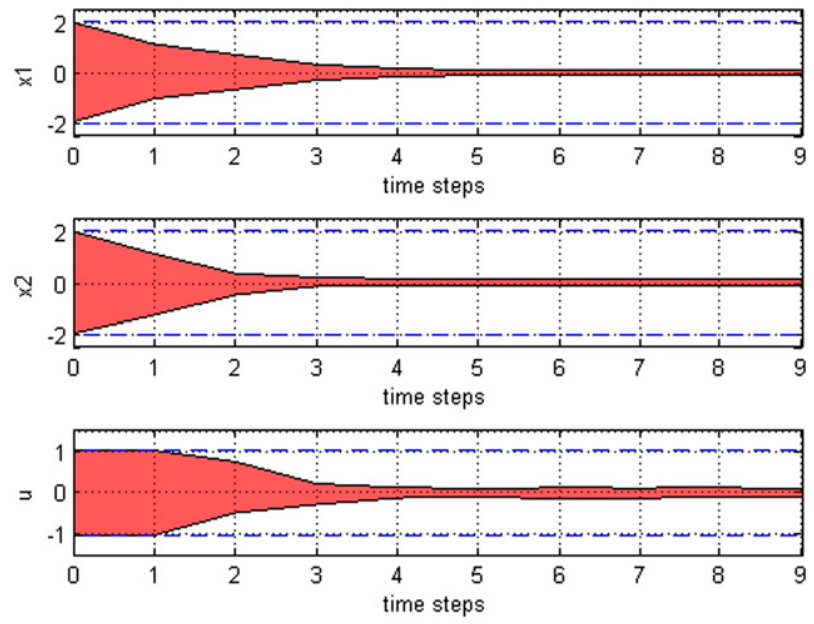

Fig. 4. Envelope of state and input trajectories over 100 simulations for REAN-MPC with 6 sensor nodes. Dashed lines denote upper and lower bounds.

and present considerations on how to extended the previous results if these assumptions are removed.

As mentioned in Section 1, the presence of delays in the network is not a crucial aspect to be explicitly considered in our framework, as they are often negligible if the sampling time is sufficiently large. Moreover, common delay compensation techniques can be implemented independently of the higher-level energy-aware control scheme, e.g., by augmenting the process model with the maximum estimated delay (Bemporad, 1998; Trimboli, Di Cairano, Bemporad, \& Kolmanovsky, 2009).

On the contrary, the presence of packet loss in the controllersensors wireless link needs to be properly addressed. Dropouts can occur in the forward channel (transmission of predictions from controller to sensors), or in the backward channel (transmission of measurements from sensors to controller). Data loss in the forward channel can be handled safely with minor modifications to the proposed control scheme. Namely, if at time $k$ the sensor nodes do not receive an updated prediction buffer as expected, they simply transmit the new measurements to the controller at the next time step $k+1$, regardless of the threshold logic. Hence, forward dropouts only affect the transmission rate over the wireless link, preserving convergence of the closed-loop system. Instead, packet dropouts in the backward channel may introduce stability issues if not properly managed. Let us focus on the case of feedback from multiple noisy sensors (exact state feedback can be handled with similar arguments). The absence of packet loss is assumed in the inner mode design (namely in (19)), in the outer mode design (definition of $Q$ in (32)), and in the state estimation algorithm (correction step (13)). In particular, if transmitted measurements can be lost, then in general $x(k)-\bar{x}(k \mid k) \in \mathcal{E}$ does not hold, and it is necessary to obtain an analogous relation in order to preserve the control scheme functioning. Assuming a maximum number of consecutive packet dropouts $p$, this can be done by imposing a communication horizon $C$, defined as the maximum allowed time interval between a correctly delivered measurement transmission and the next sensors transmission: If the controller receives the last measurements packet at time $k$, then the sensors are required to transmit updated measurements at time $k+C$ regardless of the threshold logic. Then, $C+p$ is the maximum time interval between two consecutive measurements acknowledged by the controller. Based on this time bound, it is possible to compute a set $\tilde{\mathcal{E}}$ such that $x(k)-\bar{x}(k \mid k) \in \tilde{\mathcal{E}}$, by means of an iterative algorithm where the closed-loop system is considered to receive feedback every $C+p$ time steps.

\section{Conclusions}

This paper presented an energy-aware design approach for control systems based on feedback from battery-operated wireless sensors. We investigated both a single-node scenario where exact state measurements are available, and a multiple-node scenario where measurements are affected by noise and a set of wireless nodes are used to mitigate the effects of disturbance. We proposed a novel WSN transmission strategy intended to save sensors battery by minimizing the communications over the wireless channel. This strategy is based on a threshold logic where the value of the threshold can be opportunely designed to tune the trade-off between closed-loop performance and transmission rate. Moreover, we presented a robust control scheme based on explicit model predictive control with guaranteed convergence and constraint fulfillment properties. A stronger condition of asymptotic stability, based on the results of (Lazar, Mun noz de la Pe na, Heemels, \& Alamo, 2008), is a topic that deserves further investigations. Simulation results have shown that a substantial reduction in radio utilization $(40 \%-60 \%$, which roughly corresponds to doubling the life of the wireless nodes) can be achieved with a narrow loss in system performance $(<3 \%)$.

\section{References}

Akkaya, K., \& Younis, M. (2005). A survey on routing protocols for wireless sensor networks. IEEE Transactions on Mobile Computing, 3(3), 325-349.

Akyildiz, I., Su, W., Sankarasubramaniam, Y., \& Cayirci, E. (2002). A survey on sensor networks. IEEE Communications Magazine, 40, 102-114.

Alessio, A., \& Bemporad, A. (2008). A survey on explicit model predictive control. In Proc. int. workshop on assessment and future directions of nonlinear model predictive control. Pavia, Italy.

Antsaklis, P., \& Baillieul, J. (2004). Special issue on networked control systems. IEEE Transactions on Automatic Control, 49(9).

Bemporad, A. (1998). Predictive control of teleoperated constrained systems with unbounded communication delays. In Proc. 37 th IEEE conf. on decision and control. Tampa, FL (pp. 2133-2138).

Bemporad, A., Borrelli, F., \& Morari, M. (2003). Min-max control of constrained uncertain discrete-time linear systems. IEEE Transactions on Automatic Control, 48.

Bemporad, A., \& Morari, M. (1999). Control of systems integrating logic, dynamics, and constraints. Automatica, 35(3), 407-427.

Bemporad, A., Morari, M., Dua, V., \& Pistikopoulos, E. N. (2002). The explicit linear quadratic regulator for constrained systems. Automatica, 38(1), 3-20.

Bernardini, D., \& Bemporad, A. (2008). Energy-aware robust model predictive control based on wireless sensor feedback. In Proc. 47th IEEE conf. on decision and control. Cancun, Mexico.

Bernardini, D., \& Bemporad, A. (2009). Energy-aware robust model predictive control with feedback from multiple noisy wireless sensors. In Proc. European control conf. Budapest, Hungary.

Blanchini, F. (1999). Set invariance in control-a survey. Automatica, 35(11), 1747-1768.

Blanchini, F., \& Miani, S. (2008). Set-theoretic methods in control. Basel: Birkhäuser.

Camacho, E. F., \& Bordons, C. (2004). Advanced textbooks in control and signal processing, Model predictive control (2nd ed.) London: Springer-Verlag.

Chisci, L., Garulli, A., \& Zappa, G. (1996). Recursive state bounding by parallelotopes. Automatica, 32, 1049-1055.

Di Cairano, S., \& Tseng, H. E. (2010). Driver-assist steering by active front steering and differential braking: design, implementation and experimental evaluation of a switched model predictive control approach. In Proc. 49th IEEE conf. on decision and control. Atlanta, GA (pp 2886-2891).

Feeney, L. M., \& Nilsson, M. (2001). Investigating the energy consumption of a wireless network interface in an ad hoc networking environment. In 20th annual joint conference of the IEEE computer and communications societies. Vol. 3 (pp. 1548-1557).

Jones, C. E., Sivalingam, K. M., Agrawal, P., \& Chen, J. C. (2004). A survey of energy efficient network protocols for wireless networks. Wireless Networks, 7(4), 343-358.

Kerrigan, E. C., \& Maciejowski, J. M. (2003). Robustly stable feedback min-max model predictive control. In Proc. of the American control conferenc. Vol. 4. Denver, Colorado, USA (pp. 3490-3495).

Kolmanovsky, I. V., \& Gilbert, E. G. (1998). Theory and computation of disturbance invariant sets for discrete-time linear systems. Mathematical Problems in Engineering, 4(4), 317-367.

Kumar, P. R. (2001). New technological vistas for system and control: the example of wireless networks. IEEE Control Systems Magazine, 21, 24-37.

Lazar, M., Munñoz de la Peña, D., Heemels, W. P. M. H., \& Alamo, T. (2008). On inputto-state stability of min-max nonlinear model predictive control. Systems $\mathcal{E}$ Control Letters, 57, 39-48. 
Maciejowski, J. M. (2002). Predictive control with constraints. Harlow, UK: Prentice Hall.

Milanese, M., \& Vicino, A. (1991). Optimal estimation theory for dynamic systems with set membership uncertainty: an overview. Automatica, 27(6), 997-1009.

Moteiv Corporation (2006). Tmote sky: datasheet.

Qin, S. J., \& Badgwell, T. A. (2003). A survey of industrial model predictive control technology. Control Engineering Practice, 11(7), 733-764.

Quevedo, D., Ahlén, A., \& Goodwin, G. C. (2008). Predictive power control of wireless sensor networks for closed loop control. In Proc. int. workshop on assessment and future directions of nonlinear model predictive control. Pavia, Italy.

Raghunathan, V., Pereira, C. L., Srivastava, M. B., \& Gupta, R. K. (2005). Energy aware wireless systems with adaptive power-fidelity Tradeoffs. IEEE Transactions on Very Large Scale Integration (VLSI) Systems, 13(2).

Raghunathan, V., Schurgers, C., Park, S., \& Srivastava, M. (2002). Energy aware wireless microsensor networks. IEEE Signal Processing Magazine, 19, 40-50.

Rajendran, V., Obraczka, K., \& Garcia-Luna-Aceves, J. J. (2006). Energy-efficient, collision-free medium access control for wireless sensor networks. Wireless Networks, 12(1), 63-78.

Schurgers, C. (2002). Energy-aware wireless communications. Ph.D. thesis. University of California. Los Angeles.

Scokaert, P. O. M., \& Mayne, D. Q. (1998). Min-max feedback model predictive control for constrained linear systems. IEEE Transactions on Automatic Control, $43,1136-1142$.

Sinopoli, B., Sharp, C., Schenato, L., Schaffert, S., \& Sastry, S. (2003). Distributed control applications within sensor networks. In Proc. of the IEEE special issue on distributed sensor networks. Vol. 91 (pp. 1235-1246).

Trimboli, S., Di Cairano, S., Bemporad, A., \& Kolmanovsky, I. V. (2009). Model predictive control for automotive time-delay processes: an application to airto-fuel ratio control. In Proc. 8th IFAC workshop on time delay systems. Sinaia, Romania.

Walsh, M., \& Hayes, M. (2007). A robust throughput rate control mechanism for an 802.15.4 wireless sensor network-an anti-windup approach. In American control conference (pp. 3071-3076).

Zhang, G., Chen, X., \& Chen, T. (2007). A model predictive control approach to networked systems. In Proc. of the 46th IEEE conference on decision and control. New Orleans, Louisiana, USA.

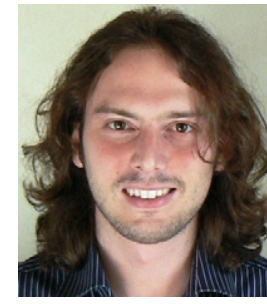

Daniele Bernardini was born in 1982. He received his Master degree in Computer Engineering in 2007 and his Ph.D. in Information Engineering in 2011, both from the University of Siena, Italy. In 2010 he was a visiting student at the Department of Electrical Engineering, Stanford University, CA. He is currently a postdoctoral research fellow at the Department of Mechanical and Structural Engineering of the University of Trento, Italy. His research interests include model predictive control, stochastic control, networked control systems and applications to energy and automotive.

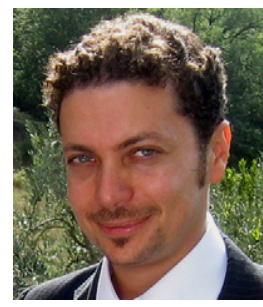

Alberto Bemporad received his master's degree in Electrical Engineering in 1993 and his Ph.D. in Contro Engineering in 1997 from the University of Florence, Italy. He spent the academic year 1996/97 at the Center for Robotics and Automation, Department of Systems Science \& Mathematics, Washington University, St. Louis, as a visiting researcher. From 1997 to 1999 he held a postdoctoral position at the Automatic Control Laboratory, ETH Zurich, Switzerland, where he collaborated as a senior researcher (2000-2002). From 1999 to 2009 he was with the Department of Information Engineering of the University of Siena, Italy, becoming an associate professor in 2005. From 2010 to 2011 he was with the Department of Mechanical and Structural Engineering of the University of Trento, Italy. Since 2011 he has been a full professor at the IMT Institute for Advanced Studies Lucca, Italy. He has published more than 220 papers in the areas of model predictive control, hybrid systems, automotive control, multiparametric optimization, computational geometry, robotics, and finance. He is the author or coauthor of various MATLAB toolboxes for model predictive contro design, including the Model Predictive Control Toolbox (The Mathworks, Inc.). He was an Associate Editor of the IEEE Transactions on Automatic Control from 2001 to 2004 and Chair of the Technical Committee on Hybrid Systems of the IEEE Control Systems Society from 2002 to 2010. He has been an IEEE Fellow since 2010. 